\title{
¿QUÉ PROFESORES NECESITA ESTA ESCUELA? NOTAS EN TORNO A LA FORMACIÓN DOCENTE UNIVERSITARIA DE CARA A LAS EXIGENCIAS QUE PLANTEA UNA ESCUELA SECUNDARIA PARA TODAS/OS
}

\author{
Noelia A. Orienti (UNLP)*
}

\begin{abstract}
Resumen
En este trabajo se pretende problematizar los modos en que la masificación del nivel medio interpela a la formación docente inicial universitaria, al demandar a los profesores el desempeño de otras funciones y plantear nuevas exigencias. Se despliegan referencias teóricas e indicios anclados en la práctica, que sugieren la necesidad de que la formación docente inicial universitaria acompañe los cambios suscitados por la nueva legislación, y prepare profesores capaces de producir dichos cambios, en la complejidad inherente a los actuales contextos socio-culturales en los que se inscriben las prácticas docentes.

En este sentido, el artículo analiza dos de las problemáticas que enfrenta la escuela secundaria a partir de su masificación: la heterogeneidad del estudiantado y las distancias generacionales; e interroga cómo estas exigencias profundizan la desigualdad al interior de las instituciones escolares, en el contexto de un modelo curricular descentralizado. Asimismo, describe algunos obstáculos que encuentra la formación universitaria de profesores para responder a las demandas actuales, e instala dos ejes de discusión para continuar la reflexión sobre este tema.
\end{abstract}

\section{Palabras clave}

Escuela secundaria - Curriculum - Profesorado - Formación docente inicial universitaria.

\footnotetext{
Especialista en Ciencias Sociales con mención en Currículum y Prácticas Escolares en Contexto (2010). FLACSO. Argentina. Profesora en Ciencias de la Educación. Facultad de Ciencias Humanas. Universidad Nacional de La Pampa. Docente en la Universidad Nacional de la Plata.
} 


\begin{abstract}
The aim of this work is to problematize the ways in wich the massification of high school brings into question the inicial college teacher training, by requiring them to fulfil other functions and present new demands. Theoretical references and evidence based in practice are displayed to suggest the need for inicial college teacher training to accompany the changes provoked by the new legislation and train teachers capable of producing such changes, in the complexity inherent to the current socio-cultural contexts in which teaching practices take part.

In this sense, the article analyzes two of the problems high school is facing from its massification: the heterogeneity of the student body and the generational gap; and questions how this requirements deepen inequality within educational institutions, in the context of a decentralized curriculum. It also describes some obstacles encountered by the college training of teachers to meet current demands, and installs two lines of discussion for further reflection on this topic.
\end{abstract}

\title{
Key words
}

High school - Curriculum - Profesorship - Initial college teacher trainin.

\section{Introducción}

A partir de la sanción de la Ley Nacional de Educación № 26.206, en el año 2.006, la obligatoriedad escolar en todo el territorio argentino se extiende hasta la finalización del nivel de la Educación Secundaria inclusive. Desde entonces, las escuelas secundarias transitan el difícil camino que las lleva de ser instituciones que históricamente seleccionaron jóvenes, a ser instituciones comprometidas con el ingreso, permanencia y egreso de las nuevas generaciones.

Como advierte Tenti Fanfani (2009), la construcción de una política efectiva excede las capacidades de los expertos y requiere de la movilización de los saberes y voluntades de los principales protagonistas del hecho educativo, es decir, los docentes. En este sentido, sostendremos aquí que no parece viable la posibilidad de cambios genuinos respecto al proyecto fundacional de la escuela secundaria, sin considerar el aporte de la formación de profesores al proceso. Pues, en efecto, así como

resulta descorazonador plantearse cambios en los sistemas formadores de profesores si el curriculum y las formas de trabajo en la escuela media no experimentan a su vez cambios sustantivos, (es) también cierto que estos cambios son improbables sin una docencia crecientemente capacitada para producirlos (Terigi, 2009:126).

Los siguientes interrogantes recorren el presente trabajo y problematizan situaciones cotidianas (1) que ofrecen algunos indicios acerca de los límites y posibilidades de la intervención de los profesores en el proceso de transfor- 
mación de la escuela secundaria: ¿Qué exigencias plantea la masividad en el nivel medio? ¿En qué condiciones se pretende que los profesores coadyuvemos en su mejora? ¿Cuál es el impacto de la descentralización educativa en las prácticas docentes bajo las restricciones actuales? ¿La formación docente inicial universitaria facilita la instauración de nuevos repertorios adecuados a los contextos y demandas? ¿Qué obstáculos encuentra la formación de profesores universitarios a la hora de ofrecer respuestas adecuadas a estos nuevos escenarios?

A riesgo de que el recorte simplifique una problemática en la que intervienen múltiples factores, el trabajo hace hincapié en demostrar la necesidad de reorganizar la formación docente inicial universitaria para afrontar la complejidad que caracteriza a los escenarios escolares contemporáneos. Partiendo de considerar algunas de las exigencias que plantea la masificación de la escuela secundaria en el contexto de un Estado débil y un modelo curricular descentralizado, pretende indagar cómo dichas exigencias profundizan la desigualdad al interior de las instituciones escolares, al demandar a los profesores el desarrollo de funciones para los cuales la formación docente inicial universitaria no los prepara. Luego, analiza algunos de los obstáculos que ésta encuentra para responder a las demandas actuales. Por último, se instalan dos ejes de discusión para continuar con la reflexión sobre esta problemática, que acaso pudieran reflejar un principio de solución en relación a la misma.

\section{Igualar homogeneizando, el proyecto de la Modernidad}

Señala Inés Dussel (2005) que más allá del "mito igualitarista", la escuela produjo (y produce) desigualdades importantes, al sostener formas de integración escolar diferenciadas que perpetuaban diferencias sociales. En parte esto se debe a que la expansión del nivel medio en nuestro país ha adoptado la misma díada que la masificación del nivel primario: igualdad-homogeneización; entendiendo igualdad no como acceso igualitario al goce de derechos políticos, civiles y sociales, sino como igualdad cultural.

La reciente instauración de la obligatoriedad escolar, conlleva necesariamente la deslegitimación de ciertos recursos que fueron constitutivos de la dinámica de este nivel de enseñanza y garantizaban excelencia. Sin embargo, aún pueden observarse continuidades con tradiciones heredadas de la gramática escolar que perduró durante todo el siglo XX. Entre los elementos de dicha matriz se destacan, siguiendo a Dussel (ibídem): la organización centralizada del nivel; el curriculum humanista enciclopédico; la disposición de las aulas; los rituales y la disciplina escolar -con sus formas rígidas, centradas en los adultos y pensadas para la formación de la élite-; la fragmentación del saber, a partir de la estructura de horarios y recreos, y la desarticulación de la propuesta formativa. 
Por otra parte, y a pesar de los cambios en las normativas y los esfuerzos de los actores involucrados, las cifras alarmantes de abandono y repitencia en el nivel medio expresan que su expansión no tuvo como consecuencia más egresados, lo cual hace impensable que la homogeneidad per se sea productora de justicia (Terigi, 2008). Estos datos tornan imperioso atender a las exigencias que comporta la masificación de la educación secundaria, ligadas al impacto en los contextos y sujetos escolares que producen las transformaciones contemporáneas.

A continuación nos centraremos en dos de las problemáticas que enfrenta el nivel a partir de la extensión de la obligatoriedad, -vinculadas con la idea de que igualar es homogeneizar- y que reclaman atención en pos de que la inclusión efectiva de todos/as no sea simple retórica. Nos estamos refiriendo a la heterogeneidad del estudiantado y a las distancias generacionales.

\section{Nuevos públicos, nuevos mandatos sociales}

Daniel Pinkasz (2009a) señala que en Argentina el crecimiento del nivel educativo medio registra un paso que va de una fase de expansión (refiriendo por tal a la comprendida aproximadamente desde fines de la década del '70 hasta 1993) a una fase de masificación (periodo que transcurre desde que se estableció con la Ley Federal de Educación la obligatoriedad del ciclo inferior de la escuela secundaria (2), hasta la actualidad). El autor sostiene que esta última etapa muestra una limitación al crecimiento de la escuela media, vinculada a las exigencias planteadas por este cambio de fase, en la que cambian los públicos y las condiciones escolares para alojarlos, en un contexto bien diferente para el cual se pensó la escuela secundaria.

Es importante no perder de vista que mientras las clases medias urbanas en ascenso -principales beneficiarias de un sistema legitimado por la meritocracia (3)-, impulsaron la etapa de expansión, cuando se inició la fase de masificación del nivel secundario los sectores medios ya habían iniciado el proceso de abandono de la escuela pública, ingresando a ésta jóvenes de grupos sociales marginados por efecto de la pobreza y la segregación urbana (Pinkasz, 2009a). Esta heterogeneidad del reclutamiento de la nueva matrícula, la presencia y sostenimiento de alumnos más débiles en su interior (Rochex, 2007), se ha convertido en uno de los problemas que presenta en el cotidiano escolar la "nueva" escuela secundaria.

El mandato de permanencia de todos/as los/as adolescentes en un nivel educativo que históricamente expulsó lo "popular", "diferente", es fuente de nuevos conflictos y desafíos (4); no sólo porque encuentra obstáculos en las tradiciones arraigadas de la cultura escolar, sino también porque se inscribe en un contexto de pobreza material y simbólica, en el cual no tienen el mismo significado las expectativas que genera el sistema educativo en términos de autorrealización y movilidad social. 
Si a comienzos del siglo XX el mandato para la escuela media estuvo relacionado con la selección y la construcción de la diferencia, y se constituyó simultáneamente en la posibilidad de asenso social, Guillermina Tiramonti (2009) sostiene que, en el actual marco de un campo educativo fragmentado (5), el mandato social de contención atraviesa a todas las instituciones escolares pero se le otorgan distintos sentidos dentro de cada grupo. En tanto para la clase media esta demanda se relaciona con un "presente gratificante", es decir, con el trato agradable, afectuoso, y un ambiente adecuado para el desarrollo de amistades; para los sectores más bajos de la población la demanda de contención está signada por una fuerte impronta reproductivista. La escuela es pensada como un "espacio de aguante", de protección de aquello que caracteriza al medio social del cual provienen los estudiantes. A modo de ejemplo, se podría señalar que es muy común que los padres de los sectores más bajos no autoricen a sus hijos a salir de la institución escolar durante las horas libres (ante la ausencia de un profesor), esgrimiendo que prefieren que estén en la escuela "sin hacer nada" a que "anden por ahí", en potenciales situaciones de riesgo, mientras ellos trabajan y no pueden controlarlos.

Por otra parte, llama la atención cómo la inclusión de "lo marginal" socaba la identidad de algunas instituciones al tiempo que el sentido de pertenencia de los profesores respecto a las mismas. Así, escuelas que eran consideradas "de excelencia" encuentran año a año un incremento en el número de estudiantes provenientes de sectores más precarizados, y esta situación comporta con frecuencia cierto malestar por parte de los docentes (sobre todo de aquellos con mayor antigüedad), quienes aducen que la inclusión de esta población a la escuela no les permite el desarrollo de su tarea con los mismos estándares de calidad. Asimismo, observan angustiados cómo las clases más acomodadas retiran a sus hijos del establecimiento, en la búsqueda de escuelas privadas que les brinden "más seguridad". También en ocasiones se vuelve frecuente, que sean los mismos docentes quienes no elijan la escuela en la que vienen desempeñando su tarea desde hace décadas para la educación de sus hijos o nietos. En este anhelo de querer volver a la "vieja y gloriosa secundaria" que con frecuencia vociferan los profesores demuestra líneas de continuidad con las tradiciones heredadas del nivel. La idea de "educar para la distinción y la jerarquía social se mantuvo presente, aún cuando las aulas se poblaron de alumnos que no pertenecían ya a la élite, y cuando las formas de distinguirse y relacionarse habían cambiado profundamente" (Dussel, 2009: 46).

Agnès van Zanten (2009), centra su análisis en el caso francés, donde también las clases medias fueron las grandes beneficiarias del proceso de expansión del nivel medio, permitiendo esta situación establecer alianzas entre padres y profesores. Afirma que en el escenario actual la relación de las familias con la escuela pública ha sido alterada significativamente por la masificación de la escuela secundaria. Las clases privilegiadas ven en la integración progresiva de jóvenes de la clase baja una amenaza, y este hecho es el que las 
lleva a pasar de una lógica de conquista a una lógica de defensa de las bases escolares de su estatus social. En función de esta lógica, los padres de clase media adoptan diversas estrategias: la deserción de las escuelas públicas con mezcla social, la intervención vigilante en las actividades de la escuela y la complementación de los aportes escolares con aportes exteriores.

Estas estrategias bien pueden ser características de las clases medias de nuestro país. Desde el registro de las experiencias personales en el campo de la educación, se observan los siguientes indicios que respaldan dicha afirmación. En primer lugar, la tendencia creciente de los padres a elegir escuelas privadas en detrimento de las públicas (socialmente heterogéneas), aún cuando las primeras sean subvencionadas y de escaso reconocimiento social. Este desplazamiento en general no se vincula directamente con la búsqueda de un mejor proceso de enseñanza- aprendizaje (que muchas veces reconocen como bueno en las escuelas públicas), sino con la preocupación de que sus hijos entablen "amistades saludables" y no se expongan a "situaciones de riesgo".

En segundo término, se observa una presencia más atenta de los padres; con frecuencia se acercan a la escuela con inquietudes frente a situaciones como las siguientes: la cantidad de inasistencias de algún profesor y la incidencia de este hecho en la calidad del aprendizaje de sus hijos; los instrumentos y criterios de evaluación empleados; la escasez de tareas que se piden realicen los chichos en horarios extraescolares o, en el otro extremo, las altas exigencias que les plantean; los modos de resolución y control de sucesos que involucran actos de violencia dentro de la institución; entre otras. Situaciones que en ciertas ocasiones develan las dificultades que tienen las instituciones de abrirse a diálogo con la comunidad educativa.

Por último, respecto a la estrategia de complementación, es ya habitual que los estudiantes de clases medias asistan a actividades relacionadas con: las artes, los idiomas, las actividades deportivas; y desde hace un tiempo, llama la atención la concurrencia de manera sistemática a clases de apoyo de aquellas asignaturas que les presentan dificultades. Lo que antes eran clases más bien esporádicas, -por ejemplo antes de un examen o ante el cierre inminente de un trimestre-, hoy en algunos casos se convierten en una modalidad paralela, durante todo el ciclo lectivo, al proceso de escolarización. Esta práctica es vista, en general, como algo altamente positivo por los profesores que consideran que las falencias de los chicos tienen su origen en los "años precedentes" y/o que las actuales condiciones de trabajo no les ofrecen posibilidades de trabajar sobre tal diversidad.

\section{Cuando la cultura escolar invisibiliza al sujeto: acerca de las dificultades del diálogo intergeneracional}

En estrecha vinculación con la problemática de la heterogeneidad en el reclutamiento de la matrícula y los múltiples sentidos que adquiere al mandato 
de contención de las nuevas generaciones, el nivel secundario afronta la problemática del desfase de su cultura y de sus modos de funcionamiento con las esferas y prácticas culturales, gustos, intereses, formas de inteligencia, de las nuevas generaciones (Rochex, op.cit.). En efecto, y aún cuando el modelo del proyecto moderno se muestra agotado, esto es, sin capacidad de responder a las nuevas escenas escolares, sus huellas persisten en el ideario docente y acentúan el enfrentamiento de dos culturas: la de los adolescentes y jóvenes y la que es propia de la tradición escolar. Al respecto, sostiene Tenti Fanfani que

\begin{abstract}
Mientras que el programa escolar tiene todavía las huellas del momento fundacional (homogeneidad, sistematicidad, continuidad, coherencia, orden y secuencia únicos, etc.), las nuevas generaciones son portadoras de culturas diversas, fragmentadas, abiertas, flexibles, móviles, inestables, etc. La experiencia escolar se convierte a menudo en una frontera donde se encuentran y enfrentan diversos universos culturales (2009: 58-59).
\end{abstract}

En este punto es importante considerar que hoy aún aquellos adolescentes (6) que provienen de los sectores sociales que históricamente accedieron a la educación secundaria son diferentes, en tanto se está configurando una "nueva adolescencia": más alejada de la cultura letrada, atravesada por la televisión e Internet, con formas de entretenimiento estructuradas en torno a las nuevas tecnologías y en espacios de socialización que, en muchos casos, se resuelven en el espacio virtual.

Asistimos también a cambios en las constituciones familiares que traen aparejados arreglos de convivencia novedosos, alteraciones en los roles de género y abren paso a nuevas figuras de la autoridad, una autoridad parental cada vez más debilitada en el seno de la familia y las instituciones (Urresti, 2009): Ios adolescentes ahora "subsocializados por sus padres, sobresocializados por medios audiovisuales blandos y estructurados como un restaurante a la carta, (...) en su proceso de socialización van eligiendo su propia aventura, como en aquellos famosos libros que tenían cincuenta finales abiertos". Esta situación enfrenta a la escuela ante un nuevo problema: al desarticularse el antiguo pacto familia-escuela, según el cual se reforzaba la autoridad de unos y otros tiene que ser construido.

Las transformaciones acaecidas en la segunda mitad del siglo XX configuraron escenarios complejos e inciertos que demandan nuevas estructuras y mandatos a la escuela. Sin embargo, la escuela parece estar en un lugar de resistencia cultural y no de apertura e intercambio con la cultura contemporánea (Tiramonti, 2005, 2009). Los discursos que circulan en las reuniones de profesores, en relación que "a los chicos de hoy no les importa nada", a la desvalorización de los códigos y estilos que construyen, los modos de protesta que ensayan, por mencionar sólo algunos, dan cuenta del abismo generacional que existe, del desconocimiento y desconfianza para con los adolescentes de esta época. 
Asimismo, aparece como una práctica extendida realizar los diagnósticos de los estudiantes remarcando sus dificultades: "no sabe", "no puede", "encuentra limitaciones", etc. Como si los adolescentes no tuvieran ninguna potencialidad que mereciera la pena subrayar, quizá inclusive como peldaño a partir del cual motivar, desarrollar, hacer emerger, las capacidades, conocimientos, saberes, estrategias que los adultos asumimos como las únicas que merecen la pena ser aprendidas (y evaluadas) y que emanan de la tradición de la cultura letrada.

En esta dirección, es relevante traer las reflexiones de Nicolazzo, quien en una investigación reciente sobre las transformaciones de la identidad de los profesores de secundaria en la provincia de Buenos Aires, señala una sensación de melancolía por la identidad perdida: "Los profesores se sienten demasiado lejos de sus alumnos, como habitantes de mundos opuestos: otros códigos culturales, otras expectativas, otras formas de entender el mundo y la vida" (2005: 123, citado en Dussel, 2009).

Haciendo foco en los discursos de los profesores, se vuelve visible cierto malestar que vincula a los jóvenes con la apatía, el desinterés, la ausencia de límites. Tal parece que estamos ni más ni menos ante la urgencia de re-construir el vínculo pedagógico, de modo que permita intercambios genuinos entre las distintas generaciones que comparten la experiencia escolar. Si queremos una escuela para todos/as, debemos comenzar a habilitar diálogos intergeneracionales, concebir a los adolecentes como transformadores y constructores de cultura, como sujetos de derecho. Porque la escuela es escenario de encuentro y desencuentro entre generaciones, el compromiso de transmitir conocimientos y generar lazos sociales no nos atañe sólo como docentes sino también como adultos (Zelmanovich, 2003).

\section{Del curriculum como proyecto al curriculum como realidad: el protagonismo del profesorado}

Las instituciones no son un puerto de llegada de normas legales o reformas, sino un lugar de constante negociación entre lo impuesto y lo practicado (Gonçalves Vidal, 2007). Somos los docentes actores privilegiados de la puesta en marcha de todo proyecto político y pedagógico, en tanto interpretamos, rediseñamos y concretamos las propuestas emanadas de las normas curriculares, e incidimos en los cambios y permanencias de las prácticas escolares.

Cierto es que las situaciones con las que nos enfrentamos a diario en el aula son diversas, complejas, impredecibles, cargadas de opciones de valor y no pueden regirse por leyes o parámetros estáticos. La actuación del docente es, en parte, siempre un acto de creación y reflexión y no la mera transferencia de opciones o modelos ideados por otros. Pero este reconocimiento no conlleva el olvido sobre el valor que representan los Diseños Curriculares, en tanto ex- 
presan la selección de cultura que debe transmitirse a las nuevas generaciones en el espacio escolar, y son el resultado de acuerdos sociales

más o menos consensuados, más amplios o más restringidos, pero en cualquier caso tienen un carácter público que trasciende lo que cada institución o docente puede resolver por sí mismo (...) El curriculum es un documento público que busca organizar y regular lo que sucede en la escuela (Dussel, 2006:3).

Laura Fumagalli (2008) explica que un contexto socio-político de fragilidad de lo público, el deterioro del imperio de la ley y el debilitamiento de la capacidad regulatoria del Estado son factores que inciden en la baja efectividad de la norma curricular en la regulación del proceso de selección del contenido de enseñanza escolar en la Argentina. Esto comporta un riesgo importante, puesto que

La existencia del diseño curricular en tanto norma escrita resulta fundamental en la educación escolar para "estar atentos a su carácter público, para conservar parámetros comunes sobre lo que se enseña la escuela, y que éstos tengan que ver con lo que se ha definido colectivamente. El currículum es una suerte de "ley" en la escuela, de norma que establece contenidos y regula lo que debe enseñarse (Dussel, 2007: 6, citado en Fumagalli, 2008)

Pinkasz (2009b) señala que las tendencias curriculares que se vienen desarrollando desde hace tres décadas impactan sobre la función de regulación del curriculum, el cual deja de considerarse como un texto acabado, para convertirse en un marco de negociación abierto. Este hecho encuentra sentido en la necesidad de contar con una revalidación periódica de los contenidos, el reconocimiento de las limitaciones del curriculum para imponer homogeneidad cultural y de los textos curriculares para ordenar las prácticas docentes, y en la necesidad de revalidar su legitimidad frente a alumnos, familias y comunidad.

Ante regulaciones externas débiles y de baja legitimidad, los establecimientos buscan constantemente un punto de equilibrio interno (Pinkasz, 2009a) y esta situación abre paso a situaciones muy heterogéneas en las instituciones, que interactúan en formas complejas con las desigualdades sociales más generales (Dussel, 2005). En efecto, es objeto de preocupación creciente el incremento de las desigualdades sociales en el interior de la escuela respecto a la apropiación de saberes y habilidades intelectuales, que en ocasiones se vincula con "dejar de lado, desconocer u "olvidar" lo que dice el curriculum que debe enseñarse, en vez de discutir y confrontar para cambiar la norma, si es que creemos que es inadecuada o injusta" (Dussel, 2006:6).

Como señalamos, la homogeneidad no brinda respuestas acertadas a la realidad actual. Pero entendemos que en la búsqueda de estrategias singulares para abordar la heterogeneidad, debemos estar atentos a los discursos 
y prácticas que en nombre de la inclusión, promueven el detrimento de la calidad educativa. Esta situación se manifiesta, como veremos, en la "poda" indiscriminada de contenidos escolares para los estudiantes provenientes de los grupos más vulnerables.

\section{Los riesgos de la autonomía escolar en las condiciones actuales}

Investigaciones recientes permiten afirmar que la relación entre el nivel de escolarización y el de las adquisiciones efectivamente realizadas cada vez es más problemático y es fuente de desigualdades crecientes entre establecimientos y entre grupos sociales. Advierte Rochex que

la concentración de alumnos de medios populares precarizados y poco familiarizados con los modos de funcionamiento y los requisitos del sistema educativo - pero también los modos de adaptación de los profesores y de los establecimientos las características y a las dificultades, reales o supuestas de los alumnos- pueden conducir a aminorar las exigencias y el tiempo consagrado a los aprendizajes y a duplicar, así, las diferencias y las desigualdades (op. cit: 70).

Parece cada vez más habitual encontrar diferencias sumamente significativas entre el desarrollo curricular que realizan los profesores en unas y otras escuelas, dependiendo de la composición social (y las expectativas) de sus alumnados. Así, mientras en unas escuelas desarrollan todos los contenidos del programa, buscan o crean materiales didácticos enriquecedores y motivan constantemente a sus estudiantes a partir de estrategias de enseñanza diversas, en otras escuelas -los mismos docentes- no contemplan las prescripciones del curriculum en relación a los contenidos mínimos, prevalece la clase magistral y el uso desmedido de un solo libro escolar que presenta una versión de los contenidos escolares casi banal.

Este tipo de adaptaciones curriculares diferenciadas que lejos de acortar distancias entre los grupos sociales las perpetúan, someten a los estudiantes de grupos sociales vulnerados y vulnerables a experiencias educativas empobrecidas, condenándolos a no poseer los saberes, estrategias y conocimientos necesarios para el ingreso exitoso a los estudios superiores y muchas veces también al ingreso y permanencia a trabajos estables y bien remunerados. Los resultados de la investigación llevada a cabo por Brito (2008, citada por Pinkasz 2009a) respecto a los efectos de la adaptación curricular en la enseñanza de la Lengua y la Literatura a los distintos grupos de estudiantes pueden leerse en esta dirección.

Para Rochex (ibídem), el crecimiento de las distancias constatadas se debe a los efectos acumulados de una segregación social creciente del espacio urbano, de la competencia y la autonomía acrecentada de los establecimientos, de las estrategias "consumistas" de las familias y de las medidas de flexibilidad, 
que introducen lógicas del mercado en la escolarización. En nuestro país, estas medidas de flexibilidad están asociadas a la descentralización administrativa del sistema educativo, que en ocasiones pregona la autonomía escolar como la panacea de todos los males. Si bien acordamos que este modelo proporciona un marco flexible en el cual desarrollar el curriculum, generando un espacio de autonomía con más protagonismo de los docentes y poder de decisión, hay que ser cautelosos y considerar en qué condiciones se delega la autogestión de las innovaciones. Como exponen Gimeno Sacristán y Pérez Gómez (1992) el diseño del curriculum basado en las instituciones sin estrategias y medios para poder ayudarles, puede llegar a ser una fórmula engañosa que, a cambio de la autonomía, pueda conducirles a una mayor responsabilidad que no supone inevitablemente mejor calidad.

Pinkazs (2009a) explica que al tiempo que mayor autonomía permite "el desarrollo por parte de un número de instituciones de estrategias de flexibilización y negociación para no someter a los sujetos a instituciones que imponen ritmos y modos de existencia para quienes las dificultades de adaptación se pagan con la expulsión", del lado de las pérdidas esta situación profundiza la desigualdad, al dejar a las instituciones a merced de sus propias posibilidades. Es decir, los beneficios de este modelo pueden opacarse si no se proveen los medios para que los actores institucionales puedan adecuarse a las exigencias que plantea la masificación. Y tal parece que los cambios curriculares de las últimas décadas pretenden implementarse sin estar acompañados de las condiciones necesarias para redundar en auténticas innovaciones que mejoren los procesos de enseñanza y aprendizaje. En tal sentido, este autor advierte que a diferencia de lo que sucedió en otros países, en Argentina las tendencias curriculares actuales resaltan la noción de autonomía, en un momento en el que se llega a la instancia de masificación sin haberse desarrollado instancias de apoyo pedagógico ni para los estudiantes para aprender a construir el oficio de alumno (por ejemplo, la instauración de la figura de tutor, la creación de cargos en los gabinetes de orientación educacional) ni para los profesores, en el sentido de ayudarles a redefinir su tarea de enseñar más allá del "dictar una materia" o "dar clases".

Los docentes conocemos bien los desafíos que esto conlleva. Adaptarnos a las nuevas exigencias que platea la desigualdad social y la masificación de la escuela secundaria no nos está resultando una tarea sencilla. Nos enfrentamos ante situaciones que vivimos como de "desborde"; estamos ante diversas demandas, nuevas funciones para las cuales la formación inicial no logra ofrecer respuestas adecuadas (7). Terigi (2009) lo explica en estos términos:

Disponer, por ejemplo, de tiempos rentados para desarrollar clases de apoyo para estudiantes que experimentan dificultades en el aprendizaje de la asignatura, implica para el profesor una reformulación de su perspectiva didáctica, para no reproducir en los tiempos 
privilegiados del apoyo las estrategia de enseñanza que no tuvieron éxito en la clase regular. Por su parte, contar con un proyecto de tutoría en la escuela implica aprender a desempeñarse en tiempos destinados a atender situaciones particulares de alumnos y familias; $y$ en tiempos para el trabajo compartido con otros profesores, con vistas a identificar a los estudiantes que afrontan problemas de inclusión, permanencia o aprendizaje. Estamos ante nuevas funciones para las cuales la formación inicial tradicional no es suficiente... (p. 133).

\section{Algunos obstáculos de la formación docente inicial universitaria para responder a las nuevas exigencias y funciones del profesorado}

Si bien el presente trabajo entiende a la formación docente como factor de transformación del nivel medio, es importante antes de continuar con esta línea de análisis, hacer dos aclaraciones. En primer término, insistimos en que la mejora de la experiencia escolar requiere ineludiblemente del compromiso de los docentes, pero de modo alguno desconocemos que una política que apueste en forma excluyente a su formación y actualización constituye una respuesta simplificadora. La posibilidad de que la obligatoriedad sea una realidad y no sólo la declaración de una ley, requiere de voluntad política, y ésta no se restringe al ámbito educativo, sino requiere políticas que articulen y atiendan en forma simultánea diversos aspectos vinculados a la calidad educativa: salud, infraestructura y equipamiento escolar, sistemas de evaluación, intensificación de la jornada escolar, diversificación de las materias, talleres y oportunidades de aprendizaje de los alumnos, trabajo colaborativo con las comunidades y sus instituciones intermedias, etc. (Vezub, 2007).

En segundo lugar, hay que atender a las condiciones laborales en las cuales se pretende que los docentes desarrollen su tarea. Los cambios son improbables si las condiciones materiales del oficio docente no acompañan la agenda política (el reconocimiento salarial, las formas de organización del trabajo docente, a modo de ejemplo).

Decíamos al inicio del trabajo que los cambios que necesita la escuela secundaria son improbables sin una docencia crecientemente capacitada para producirlos, y la formación del profesorado es una responsabilidad del Estado. Sostenemos que las actuales propuestas de formación inicial de profesores no responden a las demandas que los escenarios escolares imponen a partir de la masificación del nivel medio, lo cual no equivale a negar que se han registrado cambios, tanto en sus contenidos como en su estructura y fundamentos. Vezub (ibídem) comenta al respecto que desde hace un par de décadas la formación docente inicial y el desarrollo profesional continuo han sido ubicadas como elementos centrales de los diagnósticos educativos, foco de intervención privilegiado de las políticas de reforma y de las estrategias destinadas a la elevación de la calidad. Sin embargo, afirma, subsisten serios problemas tanto en 
el funcionamiento de las instituciones como en el perfil de sus cuerpos docentes y en la organización, planificación y conducción del subsistema formador.

A continuación, señalaremos brevemente dos ejes de debate actuales que configuran algunos de los obstáculos más importantes que encuentra la formación docente inicial universitaria a la hora de responder a las nuevas exigencias y funciones que plantea al profesorado la masificación del nivel secundario (8):

- La prioridad asignada a las disciplinas de referencia (materias disciplinarias específicas) por sobre las materias pedagógicas, "en detrimento de una formación social y cultural más amplia que posibilite a los futuros docentes sumergirse en los problemas sociales y educativos contemporáneos" (Vezub, ibídem: 10) y la organización por materias ante que proponer la interdisciplinariedad o la formación por áreas o problemas (Dussel, 2001). Dentro de las desventajas de la organización por asignaturas o materias, esta autora menciona que disminuye la posibilidad de contribuir a formar sujetos autónomos y con capacidad de adaptación a múltiples realidades.

El énfasis en las disciplinas (...) tiende a reproducir el "isomorfismo" (9) de la formación de los docentes (...), olvidando las mediaciones que efectúa la pedagogía y limitando las posibilidades de una renovación permanente que tenga en cuenta los desarrollos culturales y sociales más generales. Por último, puede advertirse que tiende a no tomar en consideración o a menospreciar toda la gama de saberes y dinámicas que configuran el trabajo docente (Dussel, ibídem: 15).

El lugar asignado a la formación pedagógica en las carreras de profesorados, tanto en relación a la carga horaria total en los planes de estudio (10), como su lugar simbólico, dan cuenta de la escasa importancia otorgada a la misma. Cuando hablamos de "lugar simbólico", nos referimos a la frecuencia con la que los profesorados universitarios asumen una separación de las disciplinas específicas respecto al resto de las asignaturas, a tal punto que se promueve la desestimación de aquellas que se denominan comúnmente "del bloque pedagógico". Las representaciones que circulan en algunas carreras sobre la escasa incidencia de éstas últimas en la formación, en ocasiones hacen que muchos estudiantes comiencen a cursarlas sin muchas expectativas, y con poco compromiso académico. Este contexto resulta poco propicio para formar docentes capaces de afrontar los desafíos de la educación más allá de los límites de "dar la materia". Aunque cabe mencionar que esta situación es bien diferente cuando se trata de estudiantes que ya están trabajando en las escuelas, los que generalmente asumen no estar lo suficientemente capacitados para analizar e intervenir ante las contingencias que todos los días se presentan en su institución o salón de clases (a modo de ejemplo: directivos que no supervisan prácticas ni ayudan a los nóveles a construir sus programas, 
construcción de formas alternativas de autoridad docente, adopción de criterios consensuados para evaluar, entre otras).

Es claro que para resolver las demandas que exige la masificación de la escuela secundaria resulta cada vez más desatinado pensar en la formación de futuros profesores sólo ponderando las disciplinas específicas. No estamos desestimando el valor de éstas en la formación, sino insistiendo en el hecho de que para llevar adelante la compleja tarea de enseñar "no basta con saber la asignatura" (11).

- El escaso valor otorgado a la práctica, que puede vislumbrarse en su escisión con la teoría, su desestimación y/o la dificultad o la falta de interés por vincularla con el subsistema para el cual los docentes se preparan, en este caso, las escuelas secundarias. Todo ello recorre el curriculum real universitario y acentúa la distancia entre la formación y las necesidades de la práctica docente derivadas del ejercicio concreto del rol (Vezub, ibídem).

Asimismo, cuando se incorpora la práctica, suele ser bajo la lógica aplicacionista (12), y se trabaja sobre situaciones simuladas del orden de lo que "debería ser" antes que de lo que realmente es. En este sentido, se suelen abordar sólo situaciones de clase e instituciones "tipo", generalmente escuelas urbanas que atienden a una población más o menos homogénea y con bajo nivel de conflictividad social. Situaciones bien diferentes a aquellas que plantea la cotidianeidad escolar a partir de la masificación de la escuela media, algunas de las cuales hemos descripto en el presente trabajo.

\section{A modo de cierre}

La masificación del nivel medio nos pone ante nuevas exigencias. Aquí nos hemos centrado en dos de ellas. Hemos analizado que una viene de la mano del mandato de inclusión de todos/as, al abrirse las puertas de la escuela a lo heterogéneo, lo diferente. La otra, es suscitada por el enfrentamiento de la cultura escolar tradicional con la cultura de las jóvenes generaciones. Ambas, merecen especial atención en pos de instalar a los jóvenes como herederos, como deudores, como un eslabón en esta cadena que nos constituye como sujetos (Kiel, 2005).

Los profesores podemos afrontar el desafío de la inclusión masiva convirtiéndonos en un factor activo de cambio, generando prácticas instituyentes, o de contrario modo, resistirnos, suscitar situaciones que provoquen desidia así como también la reiteración inconsciente de rituales que pueden convertirse en fuente de desigualdades en la experiencia escolar. En este sentido, señalamos que si bien la descentralización del sistema ha traído beneficios en el funcionamiento del curriculum, (al permitir que el curriculum se defina según la escena y los recursos de los actores que ingresan a la negociación) en las 
condiciones en que se pretende la reforma engendra algunos riesgos, entre los cuales es insoslayable atender a la profundización de la desigualdad.

No basta con que se prescriban nuevas funciones o roles para hacer frente a los desafíos que plantea la inclusión. Es necesario que el Estado interpele a los actores sociales responsables de la puesta en marcha del proyecto, genere acciones que promuevan la reflexión sobre las prácticas y la construcción de respuestas adecuadas a los nuevos escenarios escolares y sociales, para lo cual la formación docente es uno de los elementos privilegiados. Más si consideramos lo que expresa Inés Dussel (2005), respecto a que la desigualdad es un problema político y social que está en el corazón de las instituciones y las subjetividades y "no es por desconocimiento que se actúa en forma diferente con "los desiguales" sino por el reforzamiento y reiteración de dinámicas o mecanismos sociales excluyentes" (p. 2).

Esperamos haber dado cuenta de que la complejidad del trabajo docente, en el escenario de la escolaridad obligatoria, exige una formación profesional que no se agota en la disponibilidad de conocimientos disciplinares actualizados (modelo imperante), aún cuando estos resultan imprescindibles. Una propuesta de formación docente coherente con esta perspectiva debería comenzar a discutir y/o considerar diferentes cuestiones, de las cuales interesa señalar dos que creemos sustantivas por su relación con los obstáculos analizados en el punto precedente:

- la incorporación de otros contenidos a la formación vinculados, entre otros temas, a: las transformaciones socio-culturales acaecidas en las últimas décadas y su impacto en las instituciones modernas y las subjetividades, las nuevas tecnologías de la información y la comunicación (lo cual supone no sólo preparación instrumental, sino un cambio en la manera de acceder al conocimiento); la configuración de nuevas identidades juveniles; los debates en relación al curriculum y el poder, entre otros posibles y deseables.

- La formación del "prácticum" (Vezub, op. cit), lo cual supone que los docentes se apropien de los métodos y las categorías de análisis y reflexión de la práctica y puedan así proponer estrategias de intervención en función de los contextos. Esto implica que sea la carrera docente y la práctica áulica la que incida y configure la formación inicial (Legarralde, 2009), dejando de lado la lógica aplicacionista vigente. 
Conscientes de que lo expuesto configura un reto importante, entre otros aspectos porque encuentra resistencias en algunos de los sectores de la comunidad académica, estamos trabajando (reflexionando, de-construyendo, re-construyendo, imaginando, ensayando) día a día en esta dirección.

\section{Notas Bibliográficas}

(1) El trabajo de reflexión integrada recoge la experiencia profesional de la autora, como profesora del nivel medio en escuelas de la Provincia de Buenos Aires y docente de algunos de los profesorados que ofrece la Universidad Nacional de La Plata.

(2) La extensión de la edad de escolarización obligatoria, junto con otros cambios impulsados por la Reforma de la década del '90 tales como: la distinción entre secundaria inferior y superior, la postergación de la opción de modalidades especializadas, el debilitamiento de las modalidades más tradicionales y el repertorio de opciones curriculares en el ciclo superior, denotan la presencia de tendencias que comenzaron a desplegarse una década antes en los países centrales (Pinkasz, 2009).

(3) El principio de la meritocracia pretende mostrarse como aséptico, neutral, al permitir ordenar a los estudiantes de acuerdo a sus "talentos". A pesar que hace décadas se ha develado que en realidad los mismos resultan de la disponibilidad de mayor capital cultural, es posible sostener que el actual es un modelo pedagógico que sigue generando efectos de selección meritocráticos, al dejar "librados a los alumnos a sus propios recursos, con deficientes mecanismos de atención a los desajustes entre exigencias escolares y posibilidades, ocasionando en los alumnos experiencias escolares costosas, interrumpidas y en muchos casos signadas por el fracaso definitivo (Pinkasz, 2009a)".

(4) En esta dirección, puede leerse el trabajo de Orienti, N. (2009) "Escuela Secundaria Obligatoria. Los docentes ante el desafío de construir nuevas formas de disciplinamiento". Revista de la Escuela de Ciencias de la Educación, Facultad de Humanidades y Artes, Universidad Nacional de Rosario, Laborde Editor, Año 5. ISSN No 1851-6297.

(5) "Usamos la idea de fragmentación para nombrar una "distancia" que se expresa en términos de extrañeza cultural y que demarca fronteras a la pertenencia, estableciendo quiénes son los "otros" que están fuera o pertenecen a otros mundos. Cuando planteamos que el campo educativo está fragmentado hacemos referencia a distancias que no pueden medirse en términos cuantitativos (mayor o menor lejanía) ya se trate de conocimientos, capitales culturales o habilidades intelectuales, sino que se distinguen por pertenencias a mundos culturales que difieren entre sí en virtud de los valores, las expectativas y los modos de vida que los organizan" (Tiramonti, 2009).

(6) Siguiendo a Urresti (2009), cuando hacemos referencia a los adolescentes o a los jóvenes estamos hablando de un grupo de población en transición; éstos, al contrario del resto, se encuentran en la etapa del boceto, ensayando el presente ante la inminente llegada del futuro que se irá forjando con sus decisiones actuales. Los adultos han cerrado esa etapa y los niños no se han visto obligados a considerarla.

(7) Si bien aquí nos centramos en la formación inicial, en este punto vale comentar respecto a la formación docente continua, que a partir de las reformas de los años noventa, las iniciativas de perfeccionamiento se ligaron con las lógicas del mercado laboral y comenzaron a formar parte de las estrategias de competitividad, supervivencia y autorregulación de los sujetos (Vezub, 2007). Legarralde (2009) admite que por entonces, el formato de los 
sistemas masivos por cursos fue eficaz al instalar la función de capacitación como una función corriente y no excepcional de los sistemas educativos, pero tuvo como aspectos negativos la promoción del credencialismo, el fomento del individualismo en la formación continua y la escasa adecuación de la capacitación a las situaciones específicas. Estos efectos se constatan en el caso de la provincia de Buenos Aires, donde la mayoría de los docentes que realiza cursos los elige por el sencillo (y no menos peligroso) criterio de la cantidad de puntaje que otorgan.

(8) Cabe mencionar que estos rasgos que obstaculizan la formación docente que necesitamos, están en parte vinculados a la lógica academicista que prima en la formación universitaria, y por tanto dependen de estructuras y culturas que difícilmente se logren trastocar a corto plazo.

(9) El "principio de isomorfismo" supone que los profesores tienen que ser formados con una especialización y un título con una denominación equivalente a la materia que se supone tienen que enseñar" (Braslavsky, 1999, citado en Dussel, 2001). Para Terigi (2009) en la enseñanza secundaria la correspondencia entre currículos clasificados y docentes especializados quedó tempranamente establecida por el principio de designación de los profesores por especialidad, que a su vez trazó de manera precisa el sistema formador de profesores.

(10) En la mayoría de los planes de estudios de los profesorados, la formación pedagógica se compone por no más de 3-4 materias.

(11) Apropiando el título de la obra de Hernández, F. y Sancho, M. (1993) Para enseñar no basta con saber la asignatura, Barcelona, Paidós.

(12) Primero se estudian las ciencias consideradas fundantes o básicas, después las ciencias aplicadas y al final se supone que el alumno está en condiciones de "aplicar" sus conocimientos en las actividades de la práctica.

\section{Referencias Bibliográficas}

- $\quad$ Argentina. Ley 26.206. Ley de Educación Nacional. Buenos Aires, Ediciones Ocampo SRL.

- Dussel, I. (2001) La formación de docentes para la educación secundaria en América Latina: perspectivas comparadas, en C. Braslavsky, I. Dussel y P. Scaliter (eds.). Los formadores de jóvenes en América Latina. Desafíos, experiencias y propuestas. Oficina Internacional de Educación y Administración Nacional de Educación Pública del Uruguay, Ginebra, pp. 10-23.

- Dussel, I. (2005) Desigualdades sociales y desigualdades escolares en la Argentina de hoy. Algunas reflexiones y propuestas, en Tedesco. J.C. ¿Cómo superar la desigualdady la fragmentación del sistema educativo argentino? Buenos Aires: Ministerio de Educación, Ciencia y Tecnología - IIPE-UNESCO. Sede Regional Buenos Aires.

- Dussel, I. (2006) El curriculum: aproximaciones para definir: Qué debe enseñar la escuela hoy. Versión preliminar. Mimeo, Buenos Aires, FLACSO.

- Dussel, I. (2009) La escuela media y la producción de la desigualdad: continuidades y rupturas, en Tiramonti, G. y Montes, N. (comp.) La escuela media en debate. Problemas actuales y perspectivas desde la investigación, Buenos Aires, Manantial- FLACSO.

- Edelstein, G (1996) Un capítulo pendiente: el método en el debate didáctico contemporáneo, en Camilloni y otras Corrientes didácticas contemporáneas, Paidos, Buenos Aires. 
- Fumagalli, L. (2008) El Curriculum como norma pública, Clase 2, en Diploma Superior en Curriculum y Prácticas Escolares en Contexto- Cohorte 13, FLACSO-Argentina.

- Gimeno Sacristán, J. y Pérez Gómez, A. (1992) Comprender y Transformar la Enseñanza, Madrid, Morata.

- Gonçalves Vidal, D. (2007) Culturas escolares: entre la regulación y el cambio, en Revista Propuesta Educativa No 28, Buenos Aires, FLACSO.

- $\quad$ Gonçalves Vidal, D. (2008) Cultura Escolar, Clase 1, en Diploma Superior en Curriculum y Prácticas Escolares en Contexto- Cohorte 13, FLACSO-Argentina.

- Kiel, L. (2005) De sin límite a limitados, en Materiales para la Capacitación. Escuela de Capacitación CePA (Centro de Pedagogías de Anticipación), Secretaría de Educación, Ciudad de Buenos Aires.

- Legarralde, M. (2009) Los debates actuales sobre la formación docente en América Latina, Clase 10, en Especialización en Curriculum y Prácticas Escolares, FLACSOArgentina.

- Orienti, N. (2009) Escuela Secundaria Obligatoria. Los docentes ante el desafío de construir nuevas formas de disciplinamiento, en Revista de la Escuela de Ciencias de la Educación, Facultad de Humanidades y Artes, Universidad Nacional de Rosario, Laborde Editor, Año 5, ISSN N 1851-6297.

- Pinkasz, D. (1992) Los orígenes del profesorado secundario, en Birgin, A.; Braslavsky, C. (Comps.) Formación de profesores: pasado, presente y futuro. Buenos Aires, Miño y Dávila Editores.

- Pinkasz, D. (2009a) El modelo curricular mundial y la masificación de la escuela media en Argentina, Clase 7, en Especialización en Curriculum y Prácticas Escolares, FLACSO- Argentina.

- $\quad$ Pinkasz, D. (2009b) Instituciones, sujetos, forma escolar y curriculum. Un tejido selectivo de los conceptos de la Especialización, Clase 12, en Especialización en Curriculum y Prácticas Escolares, FLACSO- Argentina.

- $\quad$ Puiggrós, A. (1990) Sujetos, disciplina y curriculum, Buenos Aires, Galerna.

- Rochex, J. Y. (2007) ¿Reformar la secundaria o democratizar la escolaridad obligatoria? Problemas escolares y sociales en Francia, en Revista Mexicana de Investigación Educativa N³2, Vol. XII Enero-Marzo 2007.

- Tenti Fanfani, E. (2009) La enseñanza media hoy: masificación con exclusión social y cultural, en Tiramonti, G. y Montes, N. (comp.) La escuela media en debate. Problemas actuales y perspectivas desde la investigación, Buenos Aires, Manantial- FLACSO.

- Terigi, F. (2008) Los cambios en el formato de la escuela secundaria argentina: por qué son necesarios, por qué son tan difíciles, en Propuesta Educativa № 29, pp. 63-72.

- Terigi, F. (2009) La formación inicial de profesores de Educación Secundaria: necesidades de mejora, reconocimiento de sus límites, en Revista de Educación, 350. Septiembrediciembre 2009, pp. 123-144.

- Tiramonti, G. (2005) La escuela en la encrucijada del cambio epocal. En Educação \& Sociedade, 26, (92), p. 889-910, UNICAMP, Campinas.

- Tiramonti, G. (2009) La escuela media frente a los mandatos sociales y a las nuevas formas de diferenciación social, Clase 6, en Especialización en Curriculum y Prácticas Escolares, FLACSO- Argentina.

- Urresti, M. (2000) Cambio de escenarios sociales. Experiencia juvenil urbana y escuela, en Tenti Fanfani (Comp.), Una Escuela para los Adolescentes. Buenos Aires, Losada.

- Urresti, M. (2009) Transformaciones culturales y transiciones juveniles, Clase 5, en Especialización en Curriculum y Prácticas Escolares, FLACSO- Argentina. 
- Van Zanten, A. (2009) Las estrategias de familias de clase media ante la masificación de la escuela secundaria, en: Especialización en Curriculum y Prácticas Escolares, FLACSO- Argentina.

- Vezub, L. (2007) La formación y el desarrollo profesional docente frente a los nuevos desafíos de la escolaridad, en Revista de Currículum y formación del Profesorado, №11, Universidad de Granada.

- Zelmanovich, P. (2003) Contra el desamparo, en Enseñar Hoy. Una introducción a la Educación en tiempos de crisis. Buenos Aires, Fondo de Cultura Económica. 